\title{
Evaluating the in vitro susceptibility of bovine mastitis pathogens to a combination of kanamycin and cefalexin: Recommendations for a disk diffusion test
}

\author{
C. M. Pillar, ${ }^{* 1}$ L. Goby, $†$ D. Draghi, ${ }^{*}$ P. Grover, ${ }^{*}$ and C. Thornsberry* \\ *Eurofins Medinet, Chantilly, VA 20151 \\ †Boehringer-Ingelheim Animal Health $\mathrm{GmbH}$, Ingelheim, Germany 55216
}

\section{ABSTRACT}

Cows suffering from bovine mastitis have markedly reduced milk production because of inflammation within the udder subsequent to infection and damage from bacterial toxins. Antibiotic treatment is commonly used as a preventative and therapeutic measure for bovine mastitis. The most common pathogens include Staphylococcus aureus, various streptococci (Streptococcus dysgalactiae, Streptococcus uberis), and coliforms (Escherichia coli), which can be contracted from other infected cows or from the environment. A combination of kanamycin and cefalexin (1:1.5 wt/wt) is currently used therapeutically in Europe for the treatment of bovine mastitis, although standardized methods for the in vitro determination of the susceptibility of target pathogens have not been developed. This study evaluates the appropriate broth microdilution testing criteria for kanamycin and cefalexin administered in combination and reports the development of a disk diffusion test. At a ratio of kanamycin:cefalexin relevant to that observed in milk postadministration (10:1 wt/ wt), the minimum inhibitory concentrations were determined against 307 isolates of target mastitis pathogens (staphylococci, streptococci, and E. coli). Based on achievable concentrations in milk and the resulting distribution of minimum inhibitory concentrations, preliminary broth breakpoints for kanamycin/cefalexin (10:1 fixed ratio) of $\leq 8 / 0.8 \mu \mathrm{g} / \mathrm{mL}$ susceptible, $16 / 1.6$ $\mu \mathrm{g} / \mathrm{mL}$ intermediate, and $\geq 32 / 3.2 \mu \mathrm{g} / \mathrm{mL}$ resistant were applied to evaluated staphylococci, streptococci, and E. coli. Parallel testing by disk diffusion and resulting error-rate bounded analysis using a combined disk concentration of $30 \mu \mathrm{g}$ of kanamycin and $15 \mu \mathrm{g}$ of cefalexin resulted in the establishment of preliminary disk interpretive breakpoints of $\geq 20 \mathrm{~mm}$ susceptible, 18 to $19 \mathrm{~mm}$ intermediate, and $\leq 17 \mathrm{~mm}$ resistant for

Received April 7, 2009.

Accepted August 21, 2009.

${ }^{1}$ Corresponding author: chris.pillar@eurofinsmedinet.com staphylococci, streptococci (Strep. uberis and Strep. dysgalactiae only), and E. coli.

Key words: bovine mastitis, antibiotic combination, cefalexin, kanamycin

\section{INTRODUCTION}

Bovine mastitis is an inflammation of the mammary gland of cows caused by injury or infectious agents. The inflammatory response subsequent to infection causes an increase in SCC and damage to secretory cells adversely affecting milk production (Hortet and Seegers, 1998; Seegers et al., 2003). Common pathogens of bovine mastitis include Staphylococcus aureus, streptococci (including Streptococcus uberis, Streptococcus dysgalactiae, and Streptococcus agalactiae), and some enteric gram-negative bacilli, mainly E. coli (Watts, 1988; Bradley, 2002; Tenhagen et al., 2006). Ubrolexin (Boehringer Ingelheim, Ingelheim, Germany), a combination therapeutic consisting of kanamycin and cefalexin administered directly into the udder of infected cows in a 1:1.5 ratio (wt/wt), was licensed for use in the treatment of clinical mastitis in 21 European countries in July 2008.

The general strategy of combination therapy is to enhance the coverage against target pathogens provided by either agent alone, thus broadening the spectrum of activity of the individual agents and resulting in increased potency or synergy relative to the agents alone against target pathogens (Eliopoulos and Moellering, 1996). Combination therapy can also be useful in situations in which resistance of a particular target pathogen to one agent has emerged but susceptibility to the other agent persists.

Infection control for bovine mastitis focuses on eliminating the spread of infectious mastitis pathogens through a herd (primarily Staph. aureus, Strep. dysgalactiae, and Strep. agalactiae) and the prevention of environmental mastitis (primarily caused by $E$. coli and Strep. uberis; Bradley, 2002; LeBlanc et al., 2006). Activity against the above organisms should be a consideration when selecting the appropriate therapy. 
The combination of kanamycin and cefalexin has the benefit of increasing the coverage of either agent alone against the common mastitis pathogens and has demonstrated good field efficacy against major mastitis pathogens, mainly Staph. aureus, Strep. uberis, and E. coli (Bradley and Green, 2009). In addition, there are reports of synergy between these 2 agents against mastitis target pathogens both by time-kill kinetic analysis and reduction in MIC of the individual agents in combination relative to each agent alone (Ganiere and Denuault, 2009). However, there exists little information about how to appropriately test for the in vitro susceptibility of kanamycin and cefalexin in combination against these organisms in the context of mastitis.

It is important to take into consideration several factors when considering the appropriate in vitro testing conditions and establishment of breakpoints (interpretive criteria) of any antimicrobial agent. These factors include the pharmacokinetic activity of the drug after administration (via pharmacokinetic/pharmacodynamic modeling), the correlation of in vitro data to clinical outcome (evaluating the MIC relative to bacteriologi$\mathrm{cal} /$ clinical cure), and the in vitro activity profile of the agent against a large population of target pathogens (MIC distributions against a wild-type normal population of mastitis isolates and isolates with defined resistance patterns; CLSI, 2009a). The above data are then evaluated, and interpretive criteria (breakpoints) are set to predict the likely susceptibility or resistance of an encountered pathogen to the combination based on the resulting MIC. In contrast, current interpretive criteria for either agent alone are unlikely to reflect any enhanced potency of the agents in combination and are based on the systemic administration of these agents in humans rather than direct administration into the udder. As such, this study evaluated which ratio of the combination is appropriate for broth microdilution testing based on the availability of kanamycin and cefalexin in milk after administration of the combination. A challenge set consisting of target pathogens primarily isolated from cases of bovine mastitis was tested at the selected ratio and preliminary breakpoints were determined. This strategy takes into account the availability of either agent in milk postadministration and the resulting MIC distributions against clinical mastitis isolates and known resistant isolates, so that the proposed criteria would be relevant for the combination in the context of mastitis and allow for the detection of the emergence of resistance to the combination among targeted mastitis pathogens. Finally, a disk diffusion test that accurately reflected the activity profile of the combination in broth was developed.

\section{MATERIALS AND METHODS}

\section{Bacterial Isolates}

Isolates evaluated in this study were supplied by BioScreen Laboratory (Munster, Germany) and consisted primarily of randomly selected clinical bovine mastitis isolates from 2 European Ubrolexin registration trials (2004-2005) and recent (2009) surveillance in Europe. The data included a small subset selected primarily for resistance phenotype from the isolate repository housed at Eurofins Medinet (Chantilly, VA). The current activity profile of kanamycin and cefalexin, alone and in combination, was evaluated against 268 random bovine isolates (93\% clinical mastitis) from Europe (2004-2009; primarily originating from France, United Kingdom, and Germany) consisting of 74 E. coli, 92 Staph. aureus, and 103 streptococci (66 Strep. uberis and 37 Strep. dysgalactiae). An additional 39 isolates [27 E. coli (100\% bovine); 12 Staph. aureus (8\% bovine, $92 \%$ human)] with varied susceptibility to either kanamycin or cefalexin and resistance to both were chosen to further evaluate the ability of the selected testing conditions to detect such isolates. These isolates were selected regardless of source, date of isolation, or geographic origin, although recent bovine isolates were preferentially selected whenever possible. In all, a total of 100 E. coli, 104 Staph. aureus, and 103 Streptococcus spp. (66 Strep. uberis and 37 Strep. dysgalactiae) were evaluated.

\section{Susceptibility Testing by Broth Microdilution and Disk Diffusion}

The susceptibility of the evaluated isolates was determined in accordance with the Clinical Laboratory Standards Institute (CLSI) M31-A2 Standard (CLSI, 2009b). Susceptibility of isolates to a serial 2-fold dilution series of kanamycin $(0.06-256 \mu \mathrm{g} / \mathrm{mL})$, cefalexin (0.06-128 $\mu \mathrm{g} / \mathrm{mL})$, and kanamycin combined with cefalexin at a $10: 1$ ratio $(0.06 / 0.006-128 / 12.8 \mu \mathrm{g} / \mathrm{mL}$ kanamycin/cefalexin) was determined by broth microdilution. Panels were prepared in Mueller-Hinton broth using $10 \times$ concentrated stocks of the appropriate antimicrobials over a 2 -fold dilution series so that postinoculation, the final concentrations of the agents in the wells were at the ranges specified above. Panels were stored at $-80^{\circ} \mathrm{C}$ before inoculation. Frozen stocks of the isolates were thawed and subcultured on appropriate medium (Mueller-Hinton agar supplemented with $5 \%$ sheep blood; Remel Inc., Lenexa, KS). Colonies from these plates were then adjusted to a 0.5 McFarland standard [using demineralized water for $E$. coli 
and staphylococci, and cation-adjusted Mueller-Hinton broth (Trek Diagnostics, Cleveland, OH) for streptococci], the suspension was diluted in the appropriate testing medium (cation-adjusted Mueller-Hinton broth, supplemented with lysed horse blood for streptococci), and the panel was inoculated at a final concentration of approximately $5 \times 10^{5} \mathrm{cfu} / \mathrm{mL}$ per well. Panels were incubated at $35^{\circ} \mathrm{C}$ in ambient air, and the MIC (lowest concentration of the agent that visibly inhibited growth) was determined after 18 to $24 \mathrm{~h}$. Susceptibility of isolates to the combination was further analyzed based on their susceptibility patterns to kanamycin and cefalexin alone (CLSI, 2009b). As cephalothin is usually used to represent first-generation cephalosporins, cephalothin criteria were applied to cefalexin for the purposes of this study.

Susceptibility of the evaluated isolates by disk diffusion was assessed using disks containing $30 \mu \mathrm{g}$ of kanamycin (Oxoid, Basingstoke, United Kingdom), $30 \mu \mathrm{g}$ of cefalexin (Oxoid), and a disk containing both kanamycin and cefalexin at $30 \mu \mathrm{g}$ and $15 \mu \mathrm{g}$, respectively (Mast, Merseyside, UK). The disk content for kanamycin and cefalexin in combination was determined after an evaluation of 6 disks that varied in their concentration of either agent against a subset of resistant and susceptible target isolates (data not shown). A disk content of $30 \mu \mathrm{g}$ of kanamycin and $15 \mu \mathrm{g}$ of cefalexin was selected based on its overall correlation with broth MIC (from concurrent inocula) and the yielding of optimum disk zones for isolates presumed to be susceptible or resistant to the combination in accordance with CLSI (CLSI, 2009a). For broth microdilution, the evaluated isolates were first subcultured from frozen stocks on appropriate medium. In accordance with CLSI guidelines (CLSI, 2009b), a 0.5 McFarland of the isolate was made in $0.9 \%$ normal saline. A swab of growth from that suspension was then applied to the surface of a Mueller-Hinton agar plate (supplemented with lysed sheep blood for streptococci). After the inoculum had dried, the disks were applied to the surface of the plate, and after incubation for 16 to $20 \mathrm{~h}$ at $35^{\circ} \mathrm{C}$ in ambient air, the zones of inhibition were measured.

\section{Determination of Kanamycin and Cefalexin Residues in Milk After Administration}

Data regarding the evaluation of kanamycin and cefalexin residues in milk after administration of Ubrolexin (100,000 IU of kanamycin and $200 \mathrm{mg}$ of cefalexin; 1:1.5 wt/wt; Boehringer Ingelheim, Ingelheim, Germany) in cows was obtained from a residue study that was carried out previously by the sponsor (Boehringer Ingelheim, unpublished data). Based on the recommended dosing for the combination for the treatment of bovine mastitis (summary of product characteristics), a single intramammary dose of Ubrolexin (kanamycin:cefalexin $1: 1.5 \mathrm{wt} / \mathrm{wt}$ ) was administered per quarter after milking to each of 20 lactating Holstein-Friesian dairy cows on 2 dosing occasions with a 24-h interval. Milk was collected every $12 \mathrm{~h}$ from each animal for 9 consecutive days postadministration (excluding a subgroup of 4 animals that were killed $2 \mathrm{~d}$ postadministration). Each sample of milk was weighed and stored at $-20^{\circ} \mathrm{C}$ before assay. Marker residues of cefalexin and kanamycin A were then evaluated using suitably validated methods. For the purposes of this study, data originally presented as micrograms per kilograms of milk were converted to micrograms per milliliter (using a conversion factor of $1 \mathrm{~g}$ of milk $=1 \mathrm{~mL}$ ). Data are presented as mean concentration detected at each time point \pm standard error of the mean.

\section{Evaluation of Interpretive Criteria for Broth Microdilution and Disk Diffusion Testing}

Breakpoints for broth microdilution testing of the combination at 10:1 kanamycin:cefalexin were selected based on the MIC distributions of the combination and activity of the individual agents as they relate to the availability of kanamycin and cefalexin in milk after administration. Based on these breakpoints, disk interpretive criteria were established by constructing broth versus disk correlation scatter plots and determining the linear correlation and error rate bounding in accordance with CLSI M37-A3 by the modified Metzler and DeHaan method (CLSI, 2009a).

\section{Quality Control}

Quality control (QC) for the broth microdilution testing and disk diffusion testing of kanamycin and cefalexin individually was performed in accordance with CLSI M31-A3 with E. coli ATCC 25922, Staph. aureus ATCC 29213 (broth) and 25923 (disk) as appropriate. Preliminary QC based on the testing of 10 independent inocula of each $\mathrm{QC}$ organism against kanamycin:cefalexin 10:1 (wt/wt) by broth microdilution and against disks containing $30 \mu \mathrm{g}$ of kanamycin and $15 \mu \mathrm{g}$ of cefalexin were assessed as part of this study. Streptococcus pneumoniae ATCC 49619 was included in this portion of the analysis.

\section{RESULTS AND DISCUSSION}

To determine preliminary breakpoints for broth microdilution testing, it is important to correlate resulting MIC with clinical outcome or make them pharmacodynamically relevant. In the absence of pharmacokinetic/ 

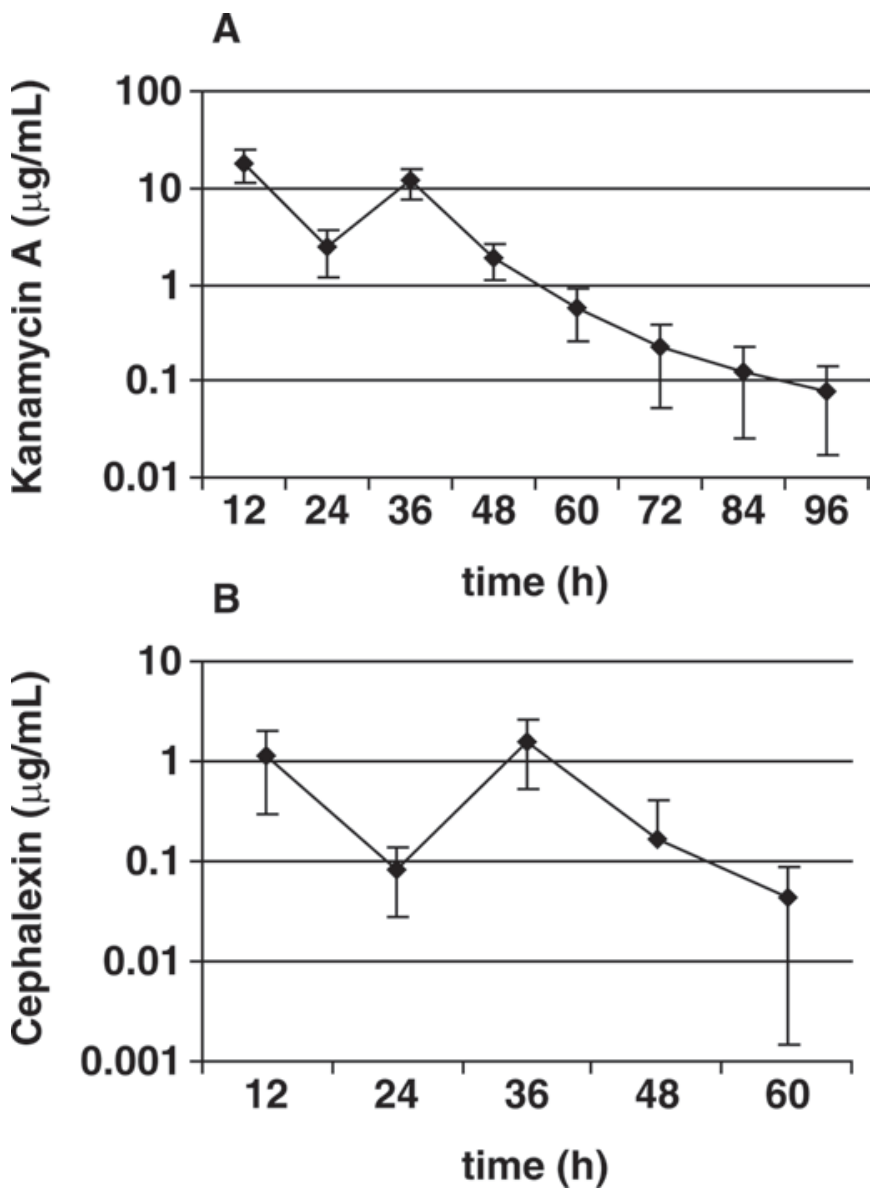

Figure 1. Detection of (A) kanamycin A and (B) cefalexin residues in the milk of dairy cows $(\mathrm{n}=20)$ after intramammary administration of Ubrolexin $(100,000 \mathrm{IU}$ of kanamycin and $200 \mathrm{mg}$ of cefalexin; 1:1.5 wt/wt; Boehringer Ingelheim, Ingelheim, Germany), with a second dose at $24 \mathrm{~h}$.

pharmacodynamic modeling data for the combination in milk, one goal of this study was to develop breakpoints that reflected the relative concentrations of both active agents in milk postadministration. The detection of residues of kanamycin $\mathrm{A}$ and cefalexin in the milk of dairy cows after administration of Ubrolexin (kanamycin:cefalexin; 1:1.5 wt/wt) over time is presented in Figure 1. The peak concentrations were detected $12 \mathrm{~h}$ after the initial dose and the second dose given at $24 \mathrm{~h}$. Kanamycin A was detected at $12 \mathrm{~h}$ at a mean of $17.9 \pm 6.6 \mu \mathrm{g} / \mathrm{mL}$, and was detected at 36 $\mathrm{h}(12 \mathrm{~h}$ after the second dose) at a mean of $11.8 \pm$ $4.1 \mu \mathrm{g} / \mathrm{mL}$. Cefalexin was detected at approximately one-tenth the concentration of kanamycin A across the dosing regimen, with $1.1 \pm 0.8 \mu \mathrm{g} / \mathrm{mL}$ detected at $12 \mathrm{~h}$ and $1.6 \pm 1.1 \mu \mathrm{g} / \mathrm{mL}$ at $36 \mathrm{~h}$. Based on the pharmacokinetics of cefalexin in relation to kanamycin in milk after administration of the combination, it was determined that a broth microdilution testing ratio of 10:1 kanamycin:cefalexin most accurately reflected the availability of these compounds postadministration $(12-48 \mathrm{~h})$ in the udders of affected cows. As such, results from testing the combination at this ratio would likely be more predictive of clinical success. Results of testing kanamycin:cefalexin at 10:1 in this study are presented below as they relate to physiologically achievable levels of kanamycin and cefalexin in milk. Broth microdilution results were then compared with those observed with a disk containing $30 \mu \mathrm{g}$ of kanamycin and $15 \mu \mathrm{g}$ of cefalexin, the disk mass selected for evaluation as described above.

The MIC activity profile of kanamycin and cefalexin at a 10:1 ratio along with the activity of either agent alone against targeted pathogens is presented in Table 1. The table lists the MIC range, modal MIC, $\mathrm{MIC}_{50}$ (concentration at which $50 \%$ of the tested isolates were inhibited), and $\mathrm{MIC}_{90}$ (concentration at which $90 \%$ of the tested isolates were inhibited) for both the normal (random clinical trial and surveillance isolates) and preferentially selected resistant (isolates preselected for resistance) populations as indicated. Disk diffusion data against the same group of isolates for kanamycin and cefalexin tested alone (both at $30 \mu \mathrm{g}$ ) along with a disk containing both kanamycin and cefalexin at $30 \mu \mathrm{g}$ and $15 \mu \mathrm{g}$, respectively, are presented in Table 2 .

Based on the overall MIC distribution of the combination against the normal and resistant populations combined, epidemiological cutoffs for susceptibility to the combination that also take into account the levels of either agent available in milk were proposed for evaluation. Based on achievable levels of either agent in milk and the separation of likely resistant and susceptible populations by MIC distribution (Figure 1), kanamycin:cefalexin (10:1) MIC breakpoints of 8/0.8 $\mu \mathrm{g} / \mathrm{mL}$ susceptible, $16 / 1.6 \mu \mathrm{g} / \mathrm{mL}$ intermediate, and $32 / 3.2 \mu \mathrm{g} / \mathrm{mL}$ resistant were evaluated for their suitability below against target pathogens.

Against the normal population of E. coli (random surveillance and clinical trial isolates, preselected resistant isolates excluded), kanamycin:cefalexin at 10:1 had a modal $\mathrm{MIC}$ and $\mathrm{MIC}_{50}$ of $2 / 0.2 \mu \mathrm{g} / \mathrm{mL}$ and $\mathrm{MIC}_{90}$ of $4 / 0.4 \mu \mathrm{g} / \mathrm{mL}$ (Figure 2). For the combination disk, a mode of $22 \mathrm{~mm}$ and mean of $21 \mathrm{~mm}$ was observed. Of the normal population, $85 \%$ were susceptible to cefalexin and $96 \%$ were susceptible to kanamycin by broth microdilution. Kanamycin-susceptible and cefalexin-nonsusceptible isolates were encountered at a rate of $12 \%$, whereas cefalexin-susceptible and kanamycin-nonsusceptible isolates were infrequent (1\%). Nonsusceptibility to both kanamycin and cefalexin was observed at a rate of $3 \%$. Susceptibility data from this population reflect current susceptibility of mastitis isolates to kanamycin and cefalexin in Europe, because 
Table 1. Susceptibility of target mastitis pathogens to kanamycin, cefalexin, and kanamycin in combination with cefalexin at 10:1 (wt/wt; Kana:Cefalex) by broth microdilution in normal and preselected populations

\begin{tabular}{|c|c|c|c|c|c|c|c|c|c|}
\hline \multirow[b]{2}{*}{ Organism } & \multirow[b]{2}{*}{ Agent } & \multicolumn{4}{|c|}{ Normal population $^{1}$} & \multicolumn{4}{|c|}{ Preselected population $^{2}$} \\
\hline & & Mode & $\mathrm{MIC}_{50}$ & $\mathrm{MIC}_{90}$ & $\% \mathrm{~S}^{3}$ & Mode & $\mathrm{MIC}_{50}$ & $\mathrm{MIC}_{90}$ & $\% \mathrm{~S}^{3}$ \\
\hline \multirow{2}{*}{ Escherichia coli } & Kanamycin & 2 & 2 & 4 & 95.9 & $>256$ & $>256$ & $>256$ & 7.4 \\
\hline & Kana:Cefalex (10:1) & $2 / 0.2$ & $2 / 0.2$ & $4 / 0.4$ & 95.9 & 64 & 64 & $>128$ & 7.4 \\
\hline Staphylococcus aureus & Cefalexin & 2 & 1 & 2 & 100.0 & 2 & 2 & 128 & 25.0 \\
\hline \multirow[t]{3}{*}{ Streptococcus spp. } & Cefalexin & 0.25 & 0.25 & 0.5 & 99.0 & & & & \\
\hline & Kanamycin & 64 & 64 & 128 & 13.6 & & & & \\
\hline & Kana:Cefalex (10:1) & $2 / 0.2$ & $2 / 0.2$ & $4 / 0.4$ & 99.0 & & & & \\
\hline \multirow[t]{3}{*}{ Streptococcus dysgalactiae } & Cefalexin & 0.25 & 0.25 & 0.5 & 97.3 & & & & \\
\hline & Kanamycin & 32 & 32 & $>256$ & 24.3 & & & & \\
\hline & Kana:Cefalex (10:1) & $4 / 0.4$ & $4 / 0.4$ & $4 / 0.4$ & 100.0 & & & & \\
\hline
\end{tabular}

${ }^{1}$ Random surveillance and clinical mastitis isolates [73 E. coli, 92 Staph. aureus, 103 Streptococcus spp. (37 Strep. dysgalactiae, 66 Strep. uberis)]. $\mathrm{MIC}_{50}$ and $\mathrm{MIC}_{90}=$ concentrations that inhibit 50 and $90 \%$ of the tested isolates, respectively.

${ }^{2}$ Preselected for resistance (27 E. coli, 12 Staph. aureus).

${ }^{3}$ Kanamycin and cefalexin interpreted according to CLSI M31-A3 (CLSI, 2009b); kanamycin and cefalexin in combination interpreted based on proposed criteria. $\mathrm{S}=$ susceptible.

this population represents a random collection of recent European clinical trial and surveillance bovine mastitis isolates. Rates for veterinary surveillance can vary considerably by country, complicating comparisons of susceptibility between surveillance studies, which are commonly geographically biased. Recent susceptibility rates reported for first-generation cephalosporins against $E$. coli mastitis isolates have ranged from $58 \%$ in Germany to 72 to $75 \%$ in the US and 84 and $97 \%$ in Finland and Israel, respectively, and for gentamicin

Table 2. Disk diffusion data of target mastitis pathogens for kanamycin, cefalexin, and $30 \mu \mathrm{g}$ of kanamycin in combination with $15 \mu \mathrm{g}$ of cefalexin (Kana:Cefalex) in normal and preselected populations

\begin{tabular}{|c|c|c|c|c|c|c|c|}
\hline Organism & Agent & \multicolumn{3}{|c|}{ Normal population $^{1}$} & \multicolumn{3}{|c|}{ Preselected population $^{2}$} \\
\hline \multirow[t]{2}{*}{ Escherichia coli } & Cefalexin & 20 & 19 & 79.5 & 6 & 15 & 48.1 \\
\hline & Kana:Cefalex (10:1) & 22 & 21 & 87.7 & 6 & 12 & 7.4 \\
\hline Staphylococcus aureus & Cefalexin & 30 & 29 & 100.0 & 6 & 11 & 25.0 \\
\hline \multirow[t]{3}{*}{ Streptococcus spp. } & Cefalexin & 30 & 31 & 100.0 & & & \\
\hline & Kanamycin & 6 & 8 & 1.0 & & & \\
\hline & Kana:Cefalex (10:1) & 28 & 30 & 100.0 & & & \\
\hline \multirow[t]{3}{*}{ Streptococcus dysgalactiae } & Cefalexin & 30 & 29 & 100.0 & & & \\
\hline & Kanamycin & 6 & 9 & 0.0 & & & \\
\hline & Kana:Cefalex (10:1) & 28 & 27 & 100.0 & & & \\
\hline
\end{tabular}

${ }^{1}$ Random surveillance and clinical mastitis isolates [73 E. coli, 92 Staph. aureus, 103 Streptococcus spp. (37 Strep. dysgalactiae, 66 Strep. uberis)].

${ }^{2}$ Preselected for resistance (27 E. coli, 12 Staph. aureus).

${ }^{3}$ Kanamycin and cefalexin interpreted according to CLSI M31-A3 (CLSI, 2009b); kanamycin and cephalexin in combination interpreted based on proposed criteria. $\mathrm{S}=$ susceptible. 


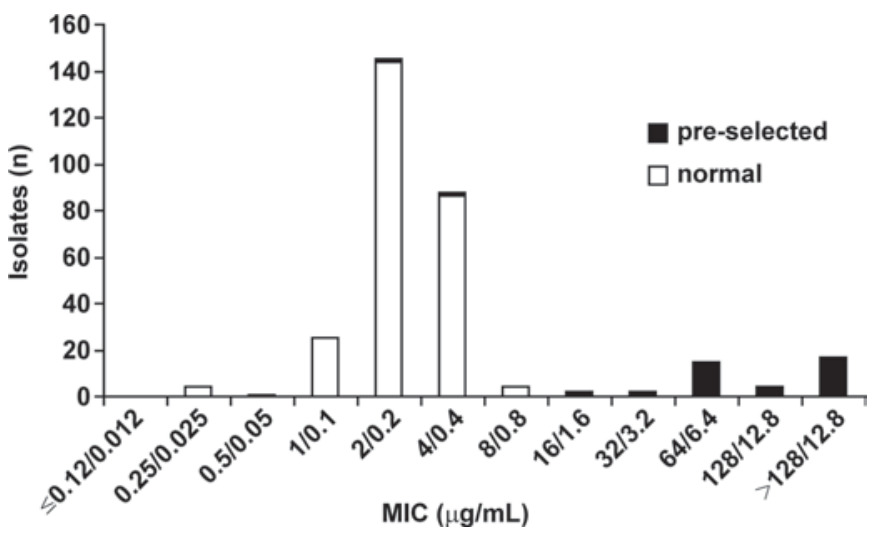

Figure 2. Distribution of MIC for kanamycin:cefalexin (10:1) against all evaluated target mastitis pathogens (normal population consisting of random surveillance/clinical trial isolates and preselected resistant population).

ranged from 98 to 100\% (Erskine et al., 2002; Lehtolainen et al., 2003; Makovec and Ruegg, 2003; Wallmann et al., 2003; White, 2006). These rates are comparable to those observed among the normal population in the current study.

Figure 3A shows the distribution of kanamycin: cefalexin 10:1 MIC against E. coli overall (normal and resistant subpopulations combined) based on cefalexin and kanamycin phenotype. Isolates susceptible to both agents had MIC $\leq 8 / 0.8 \mu \mathrm{g} / \mathrm{mL}$. One hundred percent of isolates that were susceptible to kanamycin but nonsusceptible to cefalexin also had MIC $\leq 8 / 0.8 \mu \mathrm{g} / \mathrm{mL}$ (90\% with MIC $\leq 4 / 0.4 \mu \mathrm{g} / \mathrm{mL}$ ), $100 \%$ of which had kanamycin MIC of $\leq 4 \mu \mathrm{g} / \mathrm{mL}$, well within the achievable concentration of kanamycin in milk (Figure 1A). Of isolates nonsusceptible to both kanamycin and cefalexin, $90 \%$ had MIC $\geq 128 / 12.8 \mu \mathrm{g} / \mathrm{mL}$. All isolates nonsusceptible to kanamycin but susceptible to cefalexin also had high MIC for the combination $(\geq 32 / 3.2 \mu \mathrm{g} / \mathrm{mL}$; $95 \% \geq 64 / 6.4 \mu \mathrm{g} / \mathrm{mL}$ ). Among this latter population, $50 \%$ of isolates had cefalexin MIC of $8 \mu \mathrm{g} / \mathrm{mL}$ with the remaining isolates $(50 \%)$ at $4 \mu \mathrm{g} / \mathrm{mL}$, levels of cefalexin beyond those reached in the milk (Figure 1B). As such, testing kanamycin:cefalexin at a 10:1 ratio allowed for the successful delineation of populations likely to be susceptible to the combination (those with kanamycin MIC or cefalexin MIC within the physiologically achievable range) from those unlikely to be susceptible to the combination (those with cefalexin MIC and kanamycin MIC beyond the physiologically achievable range). Figure $3 \mathrm{~B}$ shows that a similar distribution of disk zone sizes against these subpopulations was observed with the evaluated disk mass.

For the normal population of Staph. aureus, all encountered isolates were susceptible to both kanamycin and cefalexin. This finding was consistent with the low frequency of resistance $(0-4 \%)$ detected for aminoglycosides and first-generation cephalosporins among Staph. aureus bovine mastitis isolates as reported in other surveillance studies (Gentilini et al., 2000; Erskine et al., 2002; Makovec and Ruegg, 2003; Aarestrup and Schwarz, 2006). Among these isolates, a modal MIC and $\mathrm{MIC}_{50}$ of $2 / 0.2 \mu \mathrm{g} / \mathrm{mL}$ and $\mathrm{MIC}_{90}$ of $4 / 0.4 \mu \mathrm{g} / \mathrm{mL}$ was observed for kanamycin:cefalexin at 10:1. For the combination disk, a mode of $23 \mathrm{~mm}$ and mean of 26 $\mathrm{mm}$ were observed against these isolates.

Figure $4 \mathrm{~A}$ shows the distribution of kanamycin: cefalexin 10:1 MIC against Staph. aureus overall (normal and resistant subpopulations combined) based on cefalexin and kanamycin phenotypes. For the resistant population, few isolates were available for evaluation with varied susceptibility to either kanamycin or cefalexin alone. As a result, the population of resistant isolates evaluated consisted almost entirely of human isolates with resistance to both kanamycin and cefalexin. For isolates susceptible to both cefalexin and kanamycin, the MIC of kanamycin:cefalexin 10:1 were $\leq 4 / 0.4 \mu \mathrm{g} / \mathrm{mL}$ (72\% of which had MIC of $1 / 0.1-2 / 0.2$ $\mu \mathrm{g} / \mathrm{mL})$. One hundred percent of isolates nonsusceptible to both cefalexin and kanamycin had combination MIC $\geq 128 / 12.8 \mu \mathrm{g} / \mathrm{mL}$. Of the 2 isolates that were kanamycin nonsusceptible (kanamycin MIC > $256 \mu \mathrm{g}$ / $\mathrm{mL}$ ) but cefalexin susceptible, the combination had MIC of $16 / 1.6 \mu \mathrm{g} / \mathrm{mL}$, where the cefalexin MIC was $1 \mu \mathrm{g} / \mathrm{mL}$, and $32 / 3.2 \mu \mathrm{g} / \mathrm{mL}$, where the cefalexin MIC was $4 \mu \mathrm{g} / \mathrm{mL}$. As with $E$. coli, isolates likely to be susceptible to the combination based on cefalexin and kanamycin concentrations achievable in milk had low combination MIC $(\leq 4 / 0.4 \mu \mathrm{g} / \mathrm{mL})$ whereas those with cefalexin and kanamycin MIC exceeding those in milk had high combination MIC $(\geq 32 / 3.2 \mu \mathrm{g} / \mathrm{mL})$ allowing for the delineation of likely susceptible and resistant isolates. Of note, the one isolate with a cefalexin MIC of $1 \mu \mathrm{g} / \mathrm{mL}$ (susceptible, but at the limit of the achievable concentration expected in milk) and a kanamycin MIC of $>256 \mu \mathrm{g} / \mathrm{mL}$ (resistant) had an MIC of 16/1.6 $\mu \mathrm{g} / \mathrm{mL}$ to the combination, which, by the proposed criteria, would be interpreted as intermediate. Figure 4B illustrates that a similar distribution of disk zones was observed against the subpopulations of isolates as those observed by MIC.

For the evaluated streptococcal isolates, the reported activity profile represents that observed against a random normal mastitis population, because no isolates of streptococci were preselected based on resistance phenotype. Kanamycin had little appreciable activity against the evaluated species alone, with $\mathrm{MIC}_{90}$ of $>256$ and $128 \mu \mathrm{g} / \mathrm{mL}$ against Strep. dysgalactiae and Strep. uberis, respectively, although $24 \%$ of isolates of Strep. dysgalactiae were susceptible to kanamycin compared 
with $6 \%$ of Strep. uberis based on CLSI criteria (CLSI, $2009 \mathrm{~b}$ ). The activity of cefalexin alone was high against Strep. dysgalactiae and Strep. uberis with $\mathrm{MIC}_{50} / \mathrm{MIC}_{90}$ of $0.5 / 0.5 \mu \mathrm{g} / \mathrm{mL}$ and $0.25 / 0.5 \mu \mathrm{g} / \mathrm{mL}$, respectively. All streptococci were susceptible to cefalexin $(\mathrm{MIC} \leq 0.8$ $\mu \mathrm{g} / \mathrm{mL}$ ) excluding one isolate of intermediate susceptibility $(16 \mu \mathrm{g} / \mathrm{mL})$. Similar trends of high susceptibility to first-generation cephalosporins $(>97 \%)$ and low susceptibility to aminoglycosides (13-70\%), in particular among Strep. uberis, have been noted among streptococcal mastitis isolates in prior surveillance studies (Owens et al., 1990; Erskine et al., 2002; Rossitto et al., 2002).
Kanamycin:cefalexin at 10:1 had an $\mathrm{MIC}_{50}$ and $\mathrm{MIC}_{90}$ of $4 / 0.4 \mu \mathrm{g} / \mathrm{mL}$ against Strep. dysgalactiae, and an $\mathrm{MIC}_{50}$ and $\mathrm{MIC}_{90}$ of $2 / 0.2 \mu \mathrm{g} / \mathrm{mL}$ and $4 / 0.4 \mu \mathrm{g} / \mathrm{mL}$ against Strep. uberis, respectively. Overall, the combination had a mode of $28 \mathrm{~mm}$ and a mean of $27 \mathrm{~mm}$ by disk against Strep. dysgalactiae and a mode of 35 $\mathrm{mm}$ and a mean of $32 \mathrm{~mm}$ against Strep. uberis. Figure 5 A illustrates that by species, Strep. dysgalactiae and Strep. uberis have combination MIC of $\leq 8 / 0.8 \mu \mathrm{g} / \mathrm{mL}$ (excluding one isolate of Strep. uberis), $98 \%$ of which had cefalexin MIC $\leq 0.5 \mu \mathrm{g} / \mathrm{mL}$. Thus, results when testing a 10:1 ratio of kanamycin:cefalexin allow for the detection of isolates that have cefalexin and(or)
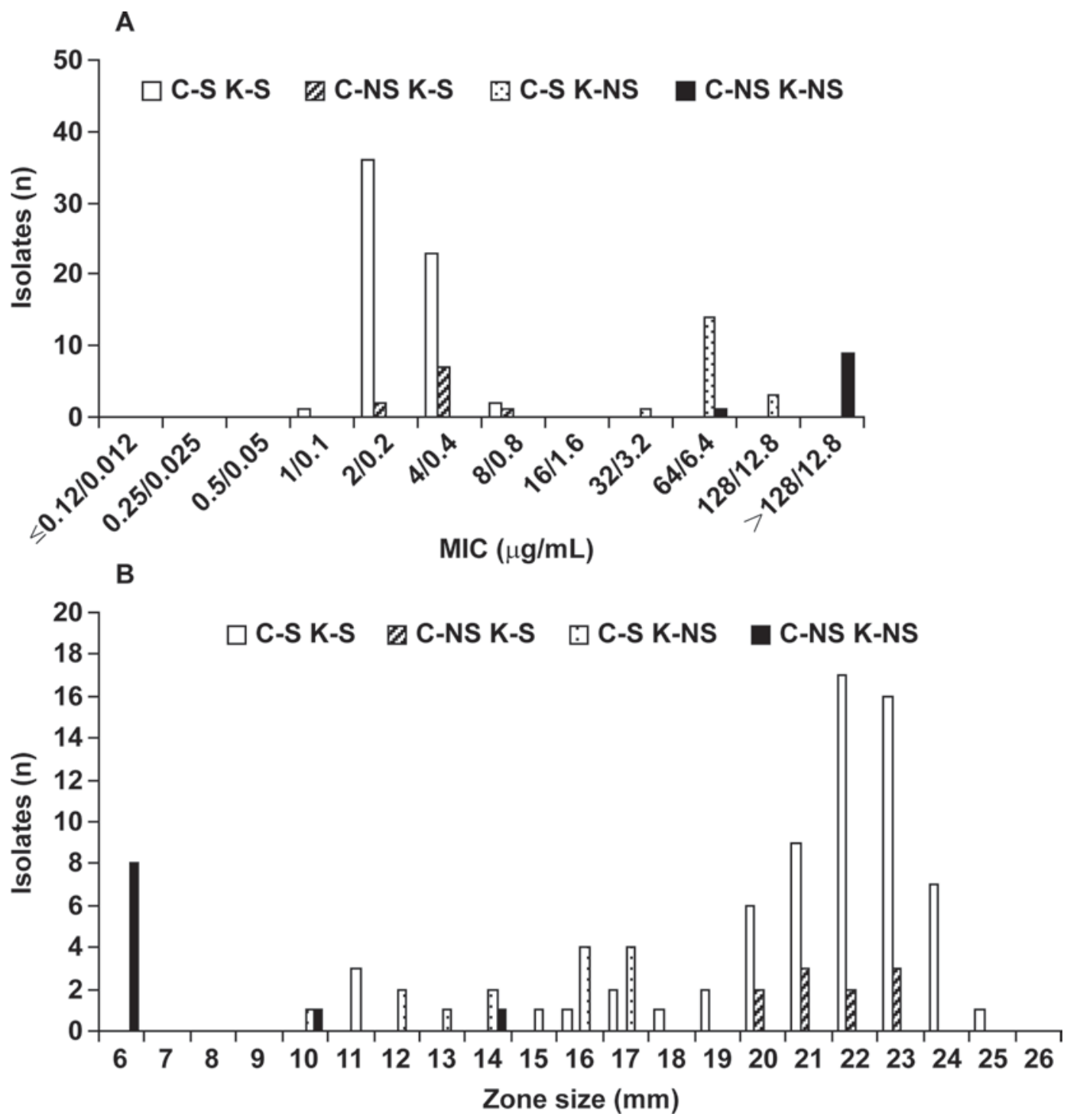

Figure 3. A) Distribution of MIC of kanamycin:cefalexin at 10:1 and B) distribution of zone sizes of kanamycin (30 $\mu \mathrm{g})$ and cefalexin (15 $\mu \mathrm{g})$ against evaluated Escherichia coli $(\mathrm{n}=100)$ based on susceptibility to kanamycin and cefalexin individually $(\mathrm{C}-\mathrm{S}=$ cefalexin susceptible, $\mathrm{C}-\mathrm{NS}=$ cefalexin nonsusceptible, K-S = kanamycin susceptible, K-NS = kanamycin nonsusceptible). 

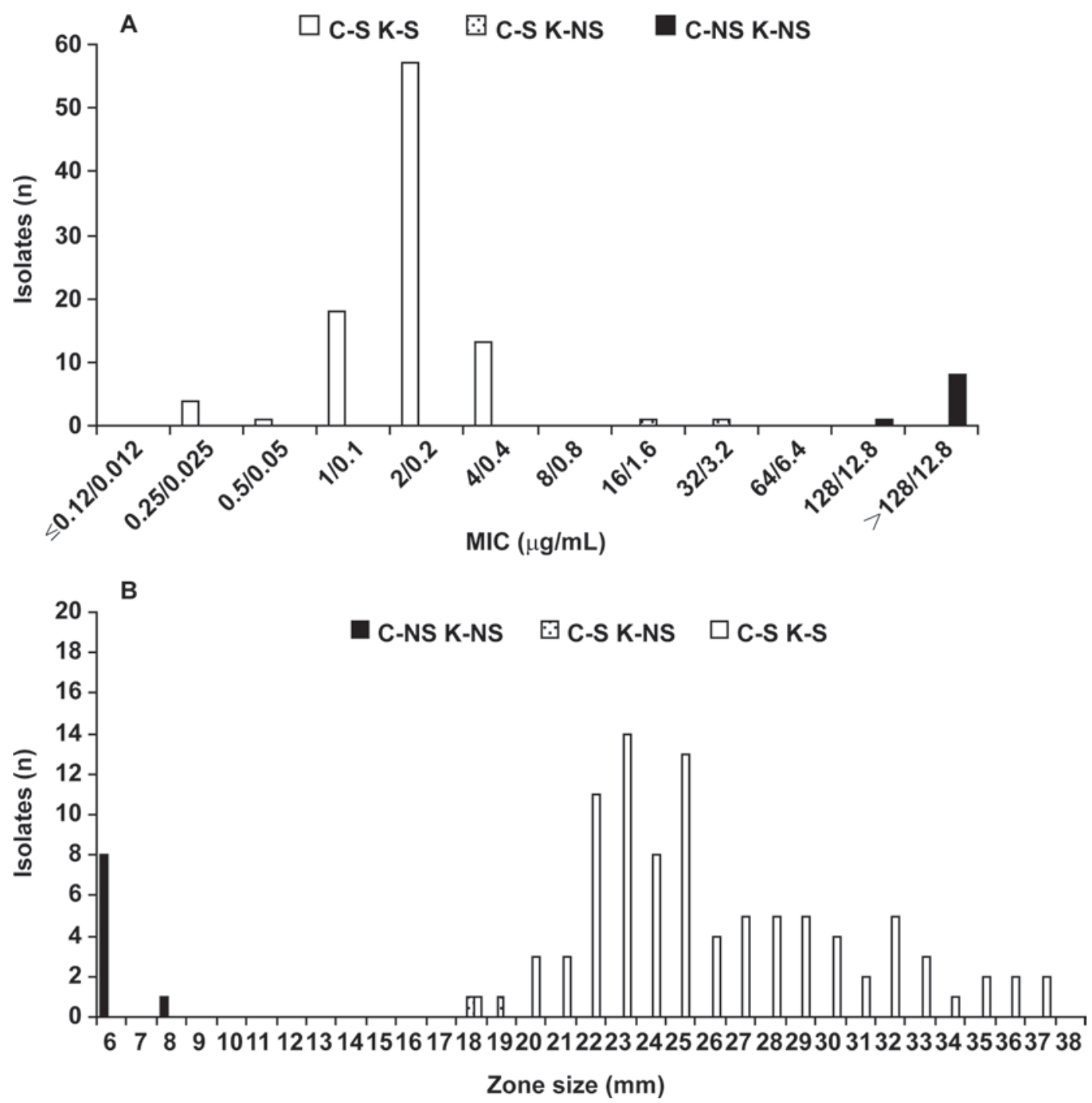

Figure 4. A) Distribution of MIC of kanamycin:cefalexin at 10:1 and B) distribution of zone sizes of kanamycin (30 $\mu \mathrm{g})$ and cefalexin (15 $\mu \mathrm{g})$ against evaluated Staphylococcus aureus $(\mathrm{n}=104)$ based on susceptibility to kanamycin and cefalexin individually $(\mathrm{C}-\mathrm{S}=$ cefalexin susceptible, $\mathrm{C}-\mathrm{NS}=$ cefalexin nonsusceptible, $\mathrm{K}-\mathrm{S}=$ kanamycin susceptible, $\mathrm{K}-\mathrm{NS}=$ kanamycin nonsusceptible).

kanamycin MIC within the achievable range in milk. For one isolate of Strep. uberis with a cefalexin MIC of $2 \mu \mathrm{g} / \mathrm{mL}$ (near the achievable concentration in milk) and a kanamycin MIC of $64 \mu \mathrm{g} / \mathrm{mL}$ (concentration beyond that achievable in milk), a combination MIC of $16 / 1.6 \mu \mathrm{g} / \mathrm{mL}$ indicative of intermediate susceptibility was observed. Figure 5B shows that the evaluated disk mass accurately reflects the MIC results observed with the combination against Strep. dysgalactiae and Strep. uberis.

The broth versus disk correlation for all evaluated isolates is depicted in Figure 6. An overall linear regression coefficient of 0.56 with a slope of -0.14 was observed. Taking into account the pharmacokinetics of cefalexin and kanamycin $\mathrm{A}$ in milk after administration and the resulting MIC distributions when testing at a pharmacokinetically relevant 10:1 ratio of kanamycin:cefalexin, preliminary broth microdilution breakpoints were set at $\leq 8 / 0.8 \mu \mathrm{g} / \mathrm{mL}$ susceptible, $16 / 1.6 \mu \mathrm{g} / \mathrm{mL}$ intermediate, and $\geq 32 / 3.2 \mu \mathrm{g} / \mathrm{mL}$ resistant. Preliminary disk breakpoints were set at $\geq 20 \mathrm{~mm}$ susceptible, 18 to $19 \mathrm{~mm}$ resistant, and $\leq 17 \mathrm{~mm}$ resistant based on minimizing overall error rates and resulting error rates lying within limits deemed acceptable by CLSI (CLSI, 2009a). Using the above criteria, only $2.0 \%$ minor errors (false intermediate by broth or disk) were observed for the evaluated disk with $1 \%$ major errors (false susceptible by disk) and no very major errors (false resistant by 

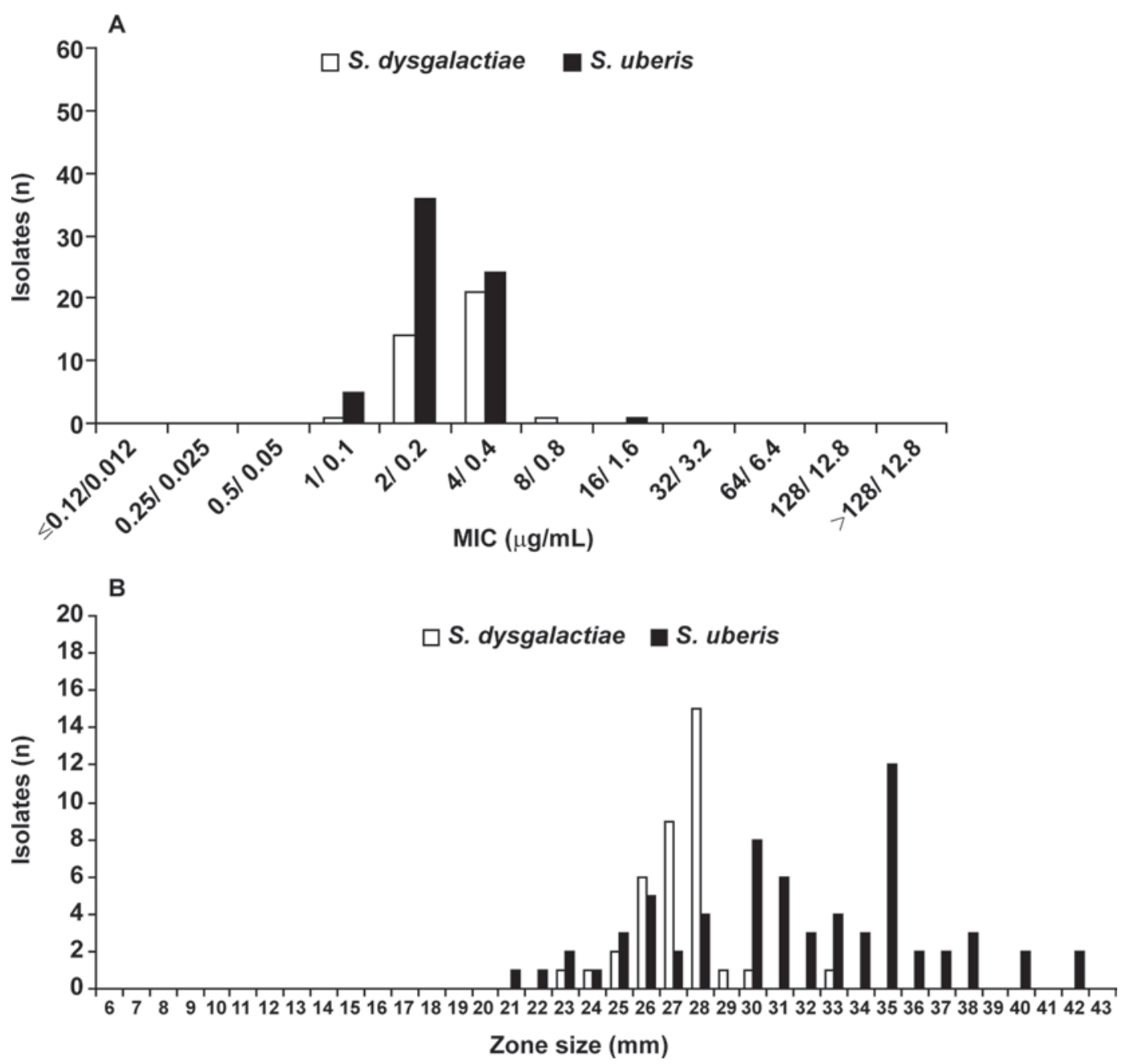

Figure 5. A) Distribution of MIC of kanamycin:cefalexin at 10:1 and B) distribution of zone sizes of kanamycin (30 $\mu \mathrm{g})$ and cefalexin (15 $\mu \mathrm{g})$ against evaluated Streptococcus spp. ( $\mathrm{n}=37$ Strep. dysgalactiae, $\mathrm{n}=66$ Strep. uberis $)$ by species.

disk). Error-rate bounding analysis as shown in Table 3 showed acceptable discrepancy rates based on CLSI criteria for the evaluated disk with the proposed criteria.

In addition, to assist in the testing of these agents in combination in other laboratories, results for the combination disk (30 $\mu \mathrm{g}$ of kanamycin, $15 \mu \mathrm{g}$ of cefalexin) and for broth microdilution (10:1 kanamycin:cefalexin) against 10 independent inocula of the relevant American Type Culture Collection QC organisms are presented in Table 4.

In conclusion, based on the concentrations of kanamycin and cefalexin after administration of Ubrolexin in dairy cows, an in vitro testing ratio of 10:1 kanamycin:cefalexin is an accurate reflection of the physiological concentration of both components after administration, and as such should be used for evaluating the in vitro susceptibility of target mastitis pathogens to kanamycin and cefalexin in combination by broth microdilution. Epidemiological breakpoints for kanamycin/cefalexin (fixed ratio 10:1) of $\leq 8 / 0.8$ $\mu \mathrm{g} / \mathrm{mL}$ susceptible, $16 / 1.6 \mu \mathrm{g} / \mathrm{mL}$ intermediate, and $32 / 3.2 \mu \mathrm{g} / \mathrm{mL}$ successfully differentiated susceptible from nonsusceptible populations among target mastitis pathogens for which the combination is indicated (Staph. aureus, Strep. uberis, Strep. dysgalactiae, and E. coli). A disk mass of $30 \mu \mathrm{g}$ of kanamycin and $15 \mu \mathrm{g}$ of cefalexin accurately reflected the broth microdilution results (kanamycin:cefalexin 10:1) observed for the evaluated target mastitis pathogens. Correlation was observed between broth and disk results and error rates were minimal and within CLSI acceptable limits when preliminary interpretive criteria of $\geq 20 \mathrm{~mm}$ susceptible, 18 to $19 \mathrm{~mm}$ intermediate, and $\leq 17 \mathrm{~mm}$ resistant were applied for the evaluated disk mass.

As the usage of antibiotics increases, so does the potential to develop resistance. Although the combina- 
Table 3. Error-rate bounding for target mastitis isolates tested against kanamycin:cefalexin at 10:1 (broth microdilution) and against $30 \mu \mathrm{g}$ of kanamycin/15 $\mu \mathrm{g}$ of cefalexin (disk diffusion)

\begin{tabular}{lcccc}
\hline & & \multicolumn{3}{c}{ Discrepancy rate, ${ }^{2} \%$} \\
\cline { 3 - 4 }${\text { MIC } \text { range }^{1}}_{$\cline { 2 - 4 }$}$ & $\mathrm{n}$ & Very major & Major & Minor \\
\hline $\mathrm{I}+2$ & 35 & 0.0 & $\mathrm{NA}^{3}$ & 0.0 \\
$\mathrm{I}+1$ to I -1 & 9 & 0.0 & 0.0 & 22.2 \\
$\leq \mathrm{I}-2$ & 263 & $\mathrm{NA}$ & 1.1 & 1.5 \\
\hline${ }^{1} \mathrm{I}=$ intermediate MIC by broth; numbers represent doubling dilutions from intermediate MIC. \\
${ }^{2}$ Very major = false resistant by disk; major = false susceptible by disk; minor = false intermediate by broth \\
or disk.
\end{tabular}

tion of cefalexin and kanamycin has the aforementioned advantages for the treatment of mastitis, the potential for multiply resistant bacteria to develop when agents are administered in combination is a concern, as is the possible effect on potentially useful classes of antibiotics in humans. In anticipation of the increased use of kanamycin in combination with cefalexin for the treatment of bovine mastitis in Europe, it becomes increasingly important to be able to monitor for any changes in resistance patterns among targeted pathogens. Although these data show that the targeted pathogens are currently largely susceptible to either or both of the agents in the combination, it is important to have testing methods in place that facilitate the detection of the emergence of resistance to either agent or both as usage increases. Furthermore, such testing would be useful for evaluating the susceptibility of the infecting pathogen to the combination, although pathogen-directed therapy is not commonly practiced in the context of mastitis, and in vitro data do not necessarily correlate with clinical outcome in this type of infection. Nonetheless, using the above preliminary criteria, the susceptibility of mastitis isolates of Staph. aureus, Strep. uberis, Strep. dysgalactiae, and E. coli to kanamycin and cefalexin in combination can be evaluated using the indicated disk mass and by broth microdilution at the indicated ratio.

\section{ACKNOWLEDGMENTS}

The authors acknowledge Christoph Keller from Bioscreen European Veterinary Disease Management Center GmbH (Münster, Germany) for supplying isolates evaluated in the current study. The study was sponsored by Boehringer-Ingelheim Animal Health GmbH (Ingelheim, Germany).

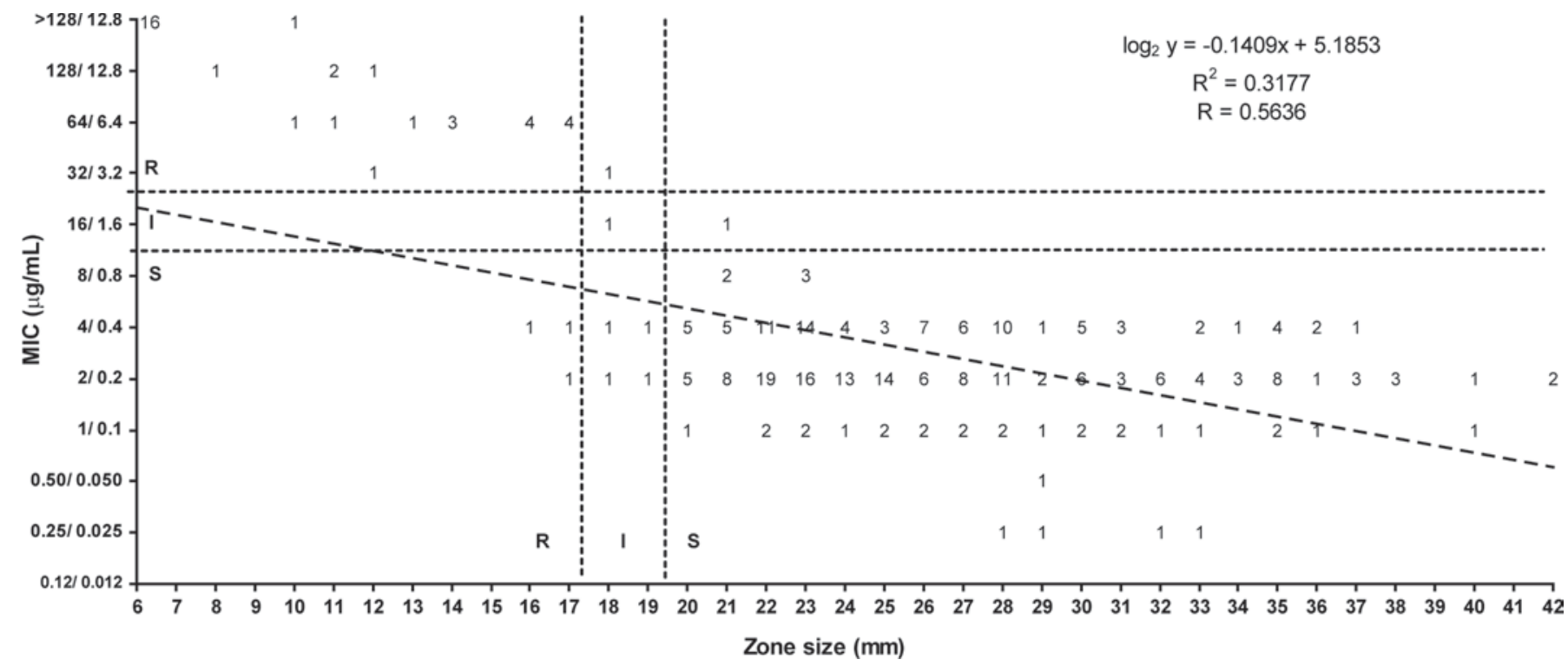

Figure 6. Broth (kanamycin:cefalexin 10:1) versus disk (kanamycin, $30 \mu \mathrm{g}$, cefalexin, $15 \mu \mathrm{g}$ ) correlation and error-rate bounding against mastitis pathogens overall $(\mathrm{n}=307)$ based on preliminary broth microdilution breakpoints of $\leq 8 / 0.8 \mu \mathrm{g} / \mathrm{mL}$ susceptible, $16 / 1.6 \mu \mathrm{g} / \mathrm{mL}$ intermediate, and $\geq 32 / 3.2 \mu \mathrm{g} / \mathrm{mL}$ resistant and preliminary disk criteria of $\geq 20 \mathrm{~mm}$ susceptible, 18 to $19 \mathrm{~mm}$ intermediate, and $\leq 17 \mathrm{~mm}$ resistant. 
Table 4. Replicate testing of kanamycin and cefalexin in combination by broth microdilution (10:1 kanamycin:cefalexin) and disk diffusion (30 $\mu \mathrm{g}$ of kanamycin, $15 \mu \mathrm{g}$ of cefalexin) against American Type Culture Collection quality control (QC) organisms

\begin{tabular}{|c|c|c|c|c|c|c|c|c|c|c|}
\hline Strain & \multicolumn{10}{|c|}{ Replicate MIC $(\mu \mathrm{g} / \mathrm{mL})$} \\
\hline Escherichia coli ATCC 25922 & $2 / 0.2$ & $2 / 0.2$ & $4 / 0.4$ & $4 / 0.4$ & $4 / 0.4$ & $4 / 0.4$ & $4 / 0.4$ & $4 / 0.4$ & $4 / 0.4$ & $4 / 0.4$ \\
\hline Staphylococcus aureus ATCC 29213 & $2 / 0.2$ & $2 / 0.2$ & $4 / 0.4$ & $2 / 0.2$ & $4 / 0.4$ & $8 / 0.8$ & $4 / 0.4$ & $2 / 0.2$ & $4 / 0.4$ & $2 / 0.2$ \\
\hline \multirow[t]{2}{*}{ Streptococcus pneumoniae ATCC 49619} & $32 / 3.2$ & $32 / 3.2$ & $16 / 1.6$ & $32 / 3.2$ & $32 / 3.2$ & $32 / 3.2$ & $32 / 3.2$ & $32 / 3.2$ & $16 / 1.6$ & $16 / 1.6$ \\
\hline & \multicolumn{10}{|c|}{ Replicate disk zone $(\mathrm{mm})$} \\
\hline \multicolumn{11}{|l|}{ Tier 1 disk QC } \\
\hline E. coli ATCC 25922 & 22 & 23 & 23 & 22 & 22 & 23 & 22 & 23 & 22 & 20 \\
\hline Staph. aureus ATCC 25923 & 24 & 24 & 24 & 24 & 24 & 24 & 24 & 25 & 22 & 20 \\
\hline Strep. pneumoniae ATCC 49619 & 15 & 16 & 18 & 18 & 17 & 17 & 18 & 16 & 18 & 17 \\
\hline
\end{tabular}

\section{REFERENCES}

Aarestrup, F., and S. Schwarz. 2006. Antimicrobial resistance in staphylococci and streptococci of animal origin. Pages 187-212 in Antimicrobial Resistance in Bacteria of Animal Origin. F. Aarestrup, ed. ASM Press, Washington, DC.

Bradley, A. 2002. Bovine mastitis: An evolving disease. Vet. J. 164:116-128.

Bradley, A. J., and M. J. Green. 2009. Factors affecting cure when treating bovine clinical mastitis with cephalosporin-based intramammary preparations. J. Dairy Sci. 92:1941-1953.

CLSI. 2009a. Development of In Vitro Susceptibility Testing Criteria and Quality Control Parameters for Veterinary Antimicrobial Agents; Approved Guideline (document M37-A3). 3rd ed. Clinical and Laboratory Standards Institute (CLSI), Wayne, PA.

CLSI. 2009b. Methods for Dilution Antimicrobial Disk and Dilution Susceptibility Tests for Bacteria Isolated from Animals; Approved Standard (document M31-A3). 3rd ed. Clinical and Laboratory Standards Institute (CLSI), Wayne, PA.

Eliopoulos, G., and R. Moellering. 1996. Antimicrobial Combinations. 4th ed. Antibiotics in Laboratory Medicine. Williams \& Wilkins, Baltimore, MD.

Erskine, R. J., R. D. Walker, C. A. Bolin, P. C. Bartlett, and D. G. White. 2002. Trends in antibacterial susceptibility of mastitis pathogens during a seven-year period. J. Dairy Sci. 85:11111118.

Ganiere, J. P., and L. Denuault. 2009. Synergistic interactions between cefalexin and kanamycin in Mueller-Hinton broth medium and in milk. J. Appl. Microbiol. 107:117-125.

Gentilini, E., G. Denamiel, P. Llorente, S. Godaly, M. Rebuelto, and O. DeGregorio. 2000. Antimicrobial susceptibility of Staphylococcus aureus isolated from bovine mastitis in Argentina. J. Dairy Sci. 83:1224-1227.

Hortet, P., and H. Seegers. 1998. Calculated milk production losses associated with elevated somatic cell counts in dairy cows: Review and critical discussion. Vet. Res. 29:497-510.
LeBlanc, S. J., K. D. Lissemore, D. F. Kelton, T. F. Duffield, and K. E. Leslie. 2006. Major advances in disease prevention in dairy cattle. J. Dairy Sci. 89:1267-1279.

Lehtolainen, T., A. Shwimmer, N. Y. Shpigel, T. Honkanen-Buzalski, and S. Pyorala. 2003. In vitro antimicrobial susceptibility of Escherichia coli isolates from clinical bovine mastitis in Finland and Israel. J. Dairy Sci. 86:3927-3932.

Makovec, J. A., and P. L. Ruegg. 2003. Antimicrobial resistance of bacteria isolated from dairy cow milk samples submitted for bacterial culture: 8,905 samples (1994-2001). J. Am. Vet. Med. Assoc. 222:1582-1589.

Owens, W. E., J. L. Watts, B. B. Greene, and C. H. Ray. 1990. Minimum inhibitory concentrations and disk diffusion zone diameter for selected antibiotics against streptococci isolated from bovine intramammary infections. J. Dairy Sci. 73:1225-1231.

Rossitto, P. V., L. Ruiz, Y. Kikuchi, K. Glenn, K. Luiz, J. L. Watts, and J. S. Cullor. 2002. Antibiotic susceptibility patterns for environmental streptococci isolated from bovine mastitis in central California dairies. J. Dairy Sci. 85:132-138.

Seegers, H., C. Fourichon, and F. Beaudeau. 2003. Production effects related to mastitis and mastitis economics in dairy cattle herds. Vet. Res. 34:475-491.

Tenhagen, B. A., G. Koster, J. Wallmann, and W. Heuwieser. 2006 Prevalence of mastitis pathogens and their resistance against antimicrobial agents in dairy cows in Brandenburg, Germany. J. Dairy Sci. 89:2542-2551.

Wallmann, J., K. Schroter, L. H. Wieler, and R. Kroker. 2003. National antibiotic resistance monitoring in veterinary pathogens from sick food-producing animals: the German programme and results from the 2001 pilot study. Int. J. Antimicrob. Agents 22:420-428.

Watts, J. L. 1988. Etiological agents of bovine mastitis. Vet. Microbiol. 16:41-66.

White, D. G. 2006. Antimicrobial resistance in pathogenic Escherichia coli from animals. Pages 145-166 in Antimicrobial resistance in bacteria of animal origin. F. Aarestrup, ed. ASM Press, Washington, DC. 